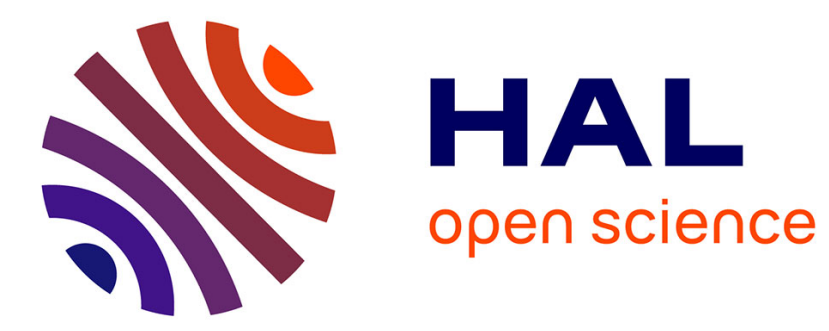

\title{
Game Theoretical Framework for Joint Channel Selection and Power Control in Hybrid NOMA
}

Amani Benamor, Oussama Habachi, Inès Kammoun, Jean-Pierre Cances

\section{To cite this version:}

Amani Benamor, Oussama Habachi, Inès Kammoun, Jean-Pierre Cances. Game Theoretical Framework for Joint Channel Selection and Power Control in Hybrid NOMA. 2020. hal-02506006

\section{HAL Id: hal-02506006 \\ https://hal.science/hal-02506006}

Preprint submitted on 12 Mar 2020

HAL is a multi-disciplinary open access archive for the deposit and dissemination of scientific research documents, whether they are published or not. The documents may come from teaching and research institutions in France or abroad, or from public or private research centers.
L'archive ouverte pluridisciplinaire HAL, est destinée au dépôt et à la diffusion de documents scientifiques de niveau recherche, publiés ou non, émanant des établissements d'enseignement et de recherche français ou étrangers, des laboratoires publics ou privés. 


\title{
Game Theoretical Framework for Joint Channel Selection and Power Control in Hybrid NOMA
}

\author{
Amani Benamor*, Oussama Habachi ${ }^{\dagger}$, Inès Kammoun* and Jean-Pierre Cances ${ }^{\dagger}$ \\ ${ }^{*}$ LETI Laboratory, National School of Engineers of Sfax, Sfax, Tunisia \\ ${ }^{\dagger}$ XLIM, University of Limoges, Limoges, France \\ Email: *amani.benamor@enis.tn, ${ }^{\dagger}$ oussama.habachi@xlim.fr, \\ *ines.kammoun@ieee.org, ${ }^{\dagger}$ jean-pierre.cances@xlim.fr
}

\begin{abstract}
Non-Orthogonal Multiple Access (NOMA) is an interesting candidate to tackle the massive access challenges in Beyond 5G (B5G) systems. However, arranging Machine Type Devices (MTDs) into NOMA clusters, and allocating resources to these clusters is a non-trivial task. In this paper, we consider a Hybrid NOMA system where every NOMA cluster is allocated an orthogonal subcarrier, and propose a game theoretical framework based on a bi-level game in order to achieve joint channel selection and power allocation for MTDs. Indeed, the proposed game is composed of a non-cooperative Power Control (PC) game underlying a cooperative Hedonic game that enables MTDs to self-organize into coalitions. Furthermore, we propose two lowcomplexity algorithms that enable us to obtain a Nash-Stable partitions where MTDs decide autonomously the resource block and the transmit power to use in order to deliver their packets.

Index Terms-Hybrid NOMA, Game theory, Resource allocation.
\end{abstract}

\section{INTRODUCTION}

Since the available frequency spectrum is absolutely limited, next generations of wireless communications should be accompanied with the adoption of non-orthogonal frequency sharing. Besides, integrating IoT devices into Beyond 5G (B5G) networks gives rise to many interesting challenges. Especially, the design of appropriate multiple access techniques is one of the most significant challenges to tackle the demands for massive connectivity and allows multiple users to share the same communication resource. Non-Orthogonal Multiple Access (NOMA) has been conceived as a breakthrough technology in the B5G because of its superior spectral efficiency. In fact, multiple NOMA users are allowed to access the same sub-carrier at the same time using either power domain multiplexing [1], [2] or code domain multiplexing [3], [4]. To this end, new Physical (PHY) and Medium Access Control (MAC) layers need to be designed to support Multiple Users Detection (MUD) technique, such as the Successive Interference Cancellation (SIC), in order to enable the receiver to separate the signals.

Indeed, even if spectrum sharing and non-orthogonal transmissions have been deeply investigated during the past decades, they are considered as the most promising multiple access techniques to be adopted since the Base Station (BS) cannot serve User Equipments (UEs) in an orthogonal manner anymore. In fact, since the advent of the $5 \mathrm{G}$ is expect- ing millions more BSs and billions of connected devices, the design of energy-efficient protocols will be even more compelling. Moreover, energy consumption at both network and terminal sides has to be optimized for economical and ecological reasons. Indeed, energy efficiency has become a key pillar in the design of communication networks. As network architecture becomes complex and the user requirement gets diverse, the role of efficient resource management has come to be a crucial task.

Several research works have investigated the design of NOMA techniques. Authors of [5], [6] and [7] proposed an uplink Power Domain-NOMA (PD-NOMA) scheme using random access scheme based on the well-known slotted Aloha protocol. In [8], the authors proposed a joint resource allocation and Power Control (PC) for random uplink NOMA based on the well-known Multi-Armed Bandit (MAB). After a training period, Machine Type Devices (MTDs) are able to determine autonomously the appropriate channel and power level for uplink transmission. Furthermore, uplink NOMA pre-allocation techniques have been considered in [9], where the authors proposed a distributed layered grant-free NOMA framework. In fact, they divided the cell into different layers based on predetermined inter-layer received power difference in order to reduce the collision probability.

PC techniques have been widely used in multi-user mobile communication systems to minimize the multi-user interference and optimize the link data rate [10]. Specifically, PC is of prime importance for uplink PD-NOMA in order to optimize the interference among users that get access to the spectrum simultaneously and to allow the receiver to separate messages using SIC. However, dynamic user pairing and power allocation using exhaustive search is not feasible because of the high computational complexity and energy cost. Henceforth, low-complexity and energy efficient distributed resource allocation algorithms are very useful for uplink PDNOMA.

As well as PC, user grouping (or clustering) is one of the potential applications and key challenges of NOMA. A cooperative game in partition formation has been proposed in [11] to design a scheme of user grouping in a NOMA system to improve the sum rate, dividing users into different coalitions and time slots are allocated to these coalitions. Ding et al. have 
studied in [12] the case of grouping two users, in a NOMAbased system, and derived some analytical insights. Obviously, considering only two users in the pairing/grouping schemes is not optimal, however it is difficult also to consider that all the users perform NOMA jointly. Hence, in order to reduce the complexity, users should be divided into groups, which will be allocated with orthogonal Resource Block (RB).

The main contributions of the paper are summarized as follows:

- We derive a new PC algorithm for MTDs in NOMA by using game theoretical framework.

- We consider a Hybrid NOMA scenario, where MTDs arrange themselves into coalitions, and each coalition transmits its packets using only one RB, in an orthogonal manner.

- We use a bi-level PC game, where MTDs use a Hedonic game to create a partition and then use a non-cooperative NOMA-based PC game to enable MTDs determining their transmit power.

The remainder of the paper is organized as follows. The next section introduces the system model and describes the signals demultiplexing using PD-NOMA. Section IIII introduces the bi-level game and proposes two low complexity algorithms to solve the joint channel selection and power allocation for NOMA networks. Before concluding the paper in Section $\mathrm{V}$. we drive in Section IV an extensive Matlab-based simulation analysis to illustrate the performance of the proposed techniques.

\section{SySTEM MODEL}

Consider a typical uplink NOMA system, depicted in Fig. 11. composed of $M$ MTDs and a BS located at the center of the cell. The MTDs are uniformly distributed in a disc with radius $r$. All MTDs are deployed in the coverage disk of the BS according to a homogeneous PPP $\Phi_{M}$ with density $\lambda_{M}$. Let us focus now on source traffic model for MTDs. We consider that MTDs operate in a regular mode until an event occurs in their environment, where they are triggered into an alarm mode. The event epicenters are represented by a homogeneous PPP $\Phi_{E}$ with density $\lambda_{E}$ in the Euclidean plane. The processes $\Phi_{M}$ and $\Phi_{E}$ are assumed independent. We choose to use PPPs because typical nodes can be reasonably assumed to be randomly deployed in the plane, in particular since we are targeting a type of transmission which does not directly involve human intervention.

Let $P_{i}^{\max }$ be the maximum transmit power for MTD $i$, and denote by $p_{i, k}$ the power allocation coefficient of MTD $i$ on the subcarrier $k$. The channel between the $i$-th MTD and the BS on the $k$-th sub-carrier is denoted by $h_{i, k}=\frac{g_{i, k}}{l_{i}}$, where $g_{i, k}$ and $l_{i}$ denote respectively the Rayleigh fading and the pathloss. The latter is modelled by Free-Space path loss model [13], i.e. $l_{i}=\left(\frac{\lambda \sqrt{G_{l}}}{5 \pi d}\right)$, where $G_{l}$ is the product of the transmit and receive antenna field radiation patterns in the Line-Of-Sight (LOS) direction, and $\lambda$ is the signal weavelength and $d$ is the

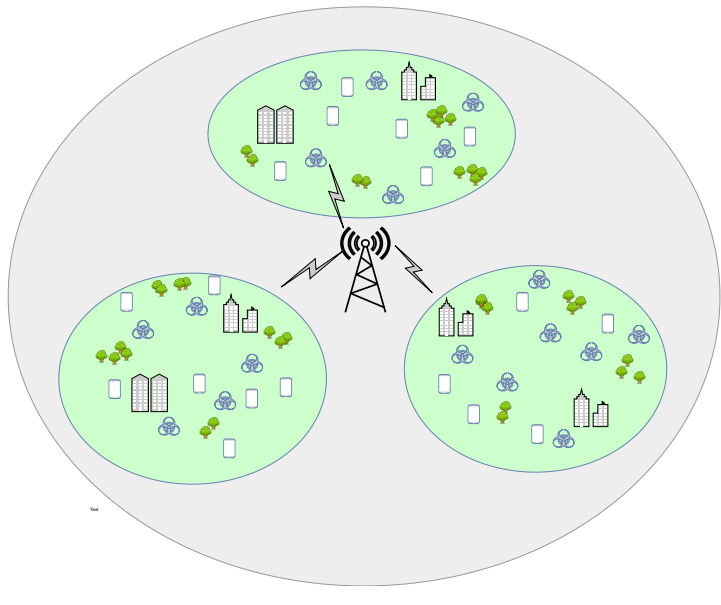

Fig. 1. The System model.

distance between MTD and BS. Hence, the received signal on the $k$-th sub-carrier at the $\mathrm{BS}$ is given by:

$$
y_{k}=\sum_{i=1}^{M} h_{i, k} \sqrt{p_{i, k}} s_{i, k}+b_{k},
$$

where $s_{i, k}$ is the transmit symbol of the MTD $i$ on the subcarrier $k$ and $b_{k}$ denotes the additive noise of variance $\sigma^{2}$ at the sub-carrier $k$. In order to split the received signal, SIC is carried out at the BS. Let us define the utility function of the MTD $i$ as follows:

$$
U_{i}\left(p_{i}\right)=\frac{\sum_{k=1}^{K} f\left(\gamma_{i, k}\right)}{\sum_{k=1}^{K} p_{i, k}},
$$

where $\gamma_{i, k}$ is the Signal-to-Interference-plus-Noise Ratio (SINR) of MTD $i$ on subcarrier $k$. The efficiency function $f(\cdot) \sqrt{1}$ reflects the probability that a packet is successfully received, and is assumed to be increasing, continuous, and S-shaped. We also require that $f(0)=0$ and $f\left(\gamma_{t h}\right)=$ $f(\infty)=1$ to ensure that when $p=0$ or $\gamma=0$ the efficiency is null. On the other hand, if the SINR is higher than the threshold $\gamma_{t h}$, the packet is successfully transmitted and then we have an efficiency of 1 . Indeed, since MTDs require high reliability and low data-rate, achieving a very high SINR is not beneficial (the packet could be transmitted successfully with lower SINR). The aforementioned utility function captures the tradeoff between throughput and battery life since its units is bits/joule. Hence, this utility function is very well suited for Wireless Sensor Network (WSN) where energy efficiency is of prime interest.

Interestingly, authors of [14], who have first introduced this utility function, have proved that the utility for a user is maximized when the user transmits only over its "best"

\footnotetext{
${ }^{1}$ Any function that satisfies the conditions mentioned above can be used. In this work, we have considered the efficiency function $f(x)=\left(1-e^{-x}\right)^{M}$ well known in the power control games where $M=100$ is the block length in bit.
} 
subcarrier, i.e. the subcarrier that requires the least amount of energy to achieve the SINR threshold $\gamma_{t h}$. Hence, we consider a hybrid NOMA system where each subcarrier is associated to a NOMA cluster, and that every MTD is associated to only one cluster. Hence, there is no interference between MTDs belonging to different NOMA clusters.

Throughout the paper, we assume that each MTD knows its Channel State Information (CSI). In Time Division Duplexing (TDD) mode, the BS can send a beacon signal at the beginning of a time slot to synchronize uplink transmissions. This beacon signal can be used as a pilot signal to allow each MTD to estimate the CSI. Due to various channel impairment (e.g., fading) and the background noise, the estimation of CSI may not be perfect. However, for simplicity, we assume that the CSI estimation is perfect in this paper. The impact of CSI estimation error on the performance needs to be studied in the future.

The BS applies then the SIC in order to separate the superimposed signals. Hence, there is an interesting question that we need to answer: how to allocate RBs and transmit power to different MTDs in order to make the BS able to separate the signals at the uplink while maximizing the capacity of the system. The same challenge should be addressed at the downlink as well. In the next section, we propose an allocation technique that addresses the aforementioned challenges.

\section{BI-LEVEL GAME THEORETICAL POWER CONTROL}

In this section, we propose a game theoretical framework composed of a Hedonic game on top of a non-cooperative PC game. In fact, MTDs are arranged into coalitions using the Hedonic game framework, then the transmit powers are determined using a non-cooperative game.

\section{A. Non-cooperative NOMA-based power control game}

Let us focus on a coalition $\mathbb{C}$ of MTDs who transmit their packets in a non-orthogonal manner using one RB. Since, we consider a hybrid NOMA system, there is no interference on the RB from MTDs that are not belonging to the coalition.

According to the SIC principle, the BS decodes the signal of the strongest MTD received on a given $\mathrm{RB}$ considering all the other MTDs of the cluster as interference, subtracts the signal of the strongest MTD from the superimposed received signal and decodes the signal of the next MTD, and so on. Hence, in order to process the SIC successfully, when considering weaker MTDs as interference, every MTD should have a Signal-to-Noise Ratio (SNR) higher than $\gamma_{t h}$. Let us now set the received SINR of each MTD in order to maximize the capacity of the NOMA network.

Proposition 1. Consider $M$ MTDs of coalition $\mathbb{C}$ are sorted using their channel in an increasing order. Consider the following target SNR vector $\Gamma=\left\{\gamma_{1}, \gamma_{2}, \cdots, \gamma_{M}\right\}$ :

$$
\begin{aligned}
\gamma_{1} & =\gamma_{t h} \\
\gamma_{2} & =\gamma_{t h} \times\left(1+\gamma_{1}\right) \\
\gamma_{3} & =\gamma_{t h} \times\left(1+\gamma_{1}+\gamma_{2}\right)
\end{aligned}
$$

If every MTD can target a distinct target SINR, the BS can process the SIC successfully.

Proof. Assume that the BS has decoded successfully the messages of the $M-1$ strongest MTDs, hence since the weakest user (MTD 1) has a target SNR of $\gamma_{t h}$, by construction, the BS can decode his message. Consider now MTD 2 (the weakest MTD that is stronger than MTD 1) and assume that the BS has decoded all the message of stronger MTDs. Hence, since the target SNR of MTD 1 is $\gamma_{t h}, p_{1}\left|h_{1}\right|^{2}=\sigma^{2} \gamma_{t h}$. Similarly, we obtain that $p_{2}\left|h_{2}\right|^{2}=\sigma^{2} \gamma_{t h}\left(1+\gamma_{t h}\right)$. Let us calculate the SINR of MTD 1 and 2 according to (3). We find out that the SINR of both MTD 1 and 2 is equal to $\gamma_{t h}$ and the BS can decode both signals. By induction, it can be easily proved that using the proposed construction, the BS can always perform the SIC successfully.

Theorem 1. Consider $M$ MTDs transmitting on the same RB using a power allocation according to Proposition 1, with a target SNR vector $\Gamma$. Then, if we allocate a new MTD with a target SNR not higher than $\max \{\Gamma\}$ the SIC will fail. Moreover, any MTD, from the allocated ones, reduces his target SNR gives rise to SIC fail.

Proof. Assume that MTD $m$ joins the coalition and targets an SNR $\gamma_{m}$, and $\exists \gamma_{i} \in \Gamma$ such that $\gamma_{i-1} \leq \gamma_{m} \leq \gamma_{i}$. Since, $p_{i}\left|h_{i}\right|^{2} /\left(\sigma^{2}+\sum_{j=1}^{i-1}\left|h_{j}\right|^{2} p_{j}\right)=\gamma_{t h}$, and $\sigma^{2}+\sum_{j=1}^{i-1}\left|h_{j}\right|^{2} p_{j}<\sigma^{2}+$ $\left|h_{m}\right|^{2} p_{m}+\sum_{j=1}^{i-1}\left|h_{j}\right|^{2} p_{j}$, the SINR of MTD $i$ becomes strictly lower than $\gamma_{t h}$ and than the decoding of the message of MTDs $i$ and all the weaker MTDs will fail. Assume now that there exists an MTD $i$ can reduce his target SNR $\gamma_{i}^{\prime}$, then we have two cases. If $\gamma_{i}^{\prime} \leq \gamma_{i-1}$, then the SIC will fail according to the first part of the proof. In fact, this case is similar to a new user who joins a coalition composed of MTDs $\{1, \cdots, i-1\}$ with a target SNR lower than $\max \left\{\gamma_{1}, \cdots, \gamma_{i-1}\right\}$. Let us focus now on the case where $\gamma_{i-1}<\gamma_{i}^{\prime}<\gamma_{i}$. Hence, we have

$$
\gamma_{i}^{\prime}=\frac{p_{i}^{\prime}\left|h_{i}\right|^{2}}{\sigma^{2}}<\frac{p_{i}\left|h_{i}\right|^{2}}{\sigma^{2}}=\frac{\sigma^{2}+\sum_{j=1}^{i-1}\left|h_{j}\right|^{2} p_{j}}{\sigma^{2}} \gamma_{t h}
$$

and the the SINR of MTD $i$ is lower than $\gamma_{t h}$ and the SIC fails.

Corollary 1. Consider a coalition $\mathbb{C}$ of MTDs transmitting on a $R B$, then we have the following allocation capacity upper bound:

$$
C_{\mathbb{C}} \leq N_{\max }^{\mathbb{C}}=\max \left\{N_{\max }^{i}\right\}
$$

where $N_{\text {max }}^{i}$ verifies $\gamma_{N_{\text {max }}^{i}} \leq \frac{P_{i}^{\max }\left|h_{i}\right|^{2}}{\sigma^{2}} \leq \gamma_{N_{\text {max }}^{i}+1}$. 
Proof. Since the highest SNR that a user can target is $\gamma_{N_{\max }}$, if every MTD can target a distinct SNR from $\Gamma$ defined in Proposition 1, $N_{\max }$ can be allocated and the BS can perform the SIC successfully. Since no MTD can reduce his target SNR, we cannot allocate more than $N_{\max }$ from $\mathbb{C}$.

1) Game formulation: Let us now formulate the power control problem using a non-cooperative game model and derive the power control iterative algorithm. Additionally, the convergence and the existence of the Nash equilibrium of this algorithm will be proved. We model the power control game with a non-cooperative game where MTDs are the players and for player $i \in \mathbb{C}$, the strategy is the couple $\left\{p_{i}, p t_{i}\right\}$ where $p_{i} \in\left[0, P_{i}^{\max }\right]$ is the transmission power of the MTD $i$, and $p t_{i} \in[0,1]$ is his transmission probability. In fact, at each time slot, if MTD $i$ has a packet to transmit, he transmits the packet in the current slot with a probability $p t_{i}$. Of course, if the system is sparse, all the MTDs will choose strategies with a transmission probability of 1 . The set of transmission power of all MTD is denoted by $\mathbf{P}$ and the set of transmission probability of all MTD is denoted by $\mathbf{P t}$. The utility function of the $i$ th MTD is defined as follows:

$$
U_{i}\left(p_{i}, \mathbf{P}_{-i}, p t_{i}, \mathbf{P t}_{-i}\right)=p t_{i} \frac{f\left(\gamma_{i}\right)}{p_{i}},
$$

where $\mathbf{P}_{-i}$ and $\mathbf{P} \mathbf{t}_{-i}$ are the transmit power and probability of MTDs other than $i$ respectively.

The design of the utility function is of prime importance in game theory. It reflects the desired goal of the game. In this paper, the objectives of the devices is to satisfy the SINR requirement and to reduce the power consumption as much as possible. The proposed utility function, that has bits per joule as units, perfectly captures the tradeoff between throughput and battery life and is particularly suitable for applications where energy efficiency is crucial. The increasing, continuous and S-shaped "efficiency" function $f(\cdot)$ measures the packet success rate. Of course, there is no utility for the devices if the BS fails decoding his signal when performing the SIC.

The Nash equilibrium, which is a solution concept for noncooperative game, is achieved when no player can increase his utility by deviating from the equilibrium unilaterally. Therefore, the Nash equilibrium $\left\{\mathbf{P}^{*}, \mathbf{P t}^{*}\right\}$ satisfies:

$$
\begin{gathered}
U_{i}\left(p_{i}^{*}, \mathbf{P}_{-i}^{*}, p t_{i}^{*}, \mathbf{P t}_{-i}^{*}\right) \geq U_{i}\left(p_{i}, \mathbf{P}_{-i}^{*}, p t_{i}, \mathbf{P t}_{-i}^{*}\right) \\
\forall\left\{p_{i}, p t_{i}\right\}, i \in \mathbb{C} .
\end{gathered}
$$

2) Power control algorithm: In this section, we propose a power control algorithm that determines, for each device of the coalition, the transmit power as well as the transmission probability. Note that this algorithm only requires the CSI of coalition's members and thus the power control can be processed in a distributed manner. If it is impossible for each device in the coalition to target a distinct target SNR, we use a layered system where at each layer only one MTD can join the group of devices targeting the same SNR. Moreover, since each device targets the lowest SNR that is not already taken in the current layer, we ensure that the proposed power control algorithm is energy efficient. The proposed power control algorithm is detailed in Algorithm 1 .

Algorithm 1 Power Control Algorithm

Input: the set of devices in the coalition $\mathbb{C}$, and the channels $\mathbf{H}(k, \mathbb{C})=\left(h_{1, k}, \ldots, h_{|\mathbb{C}|, k}\right)$ of MTD in the RB $k$

Output: a power vector $\mathbf{p}$ and a transmission probability vector pt

Initialization: $\Gamma=\left\{\gamma_{1}, \gamma_{2}, \cdots, \gamma_{N_{\max }}\right\}$. Sort $\mathbf{H}(k, \mathbb{C})$ using second coordinate in an increasing order obtaining vectors $\Pi$ and $\left(h_{1, k}^{\prime}, \ldots, h_{|\mathbb{C}|, k}^{\prime}\right)$

$\mathbf{p}=0_{1 \times|\mathbb{C}|}, \mathbf{p t}=0_{1 \times|\mathbb{C}|}$ and $\Lambda=0_{N_{\max } \times|\mathbb{C}|}$ is the allocation vector.

$$
\begin{aligned}
& \text { for } i=1:|\mathbb{C}| \mathbf{d o} \\
& m=\max _{j}\left\{\frac{f\left(\gamma_{j}\right) \times\left|h_{i, j}\right|^{2}}{\|\Lambda(m,:)\|_{0} \times \gamma_{j}} \mid j \leq N_{\text {max }}^{i}\right\} \\
& \Lambda\left(m,\left.\|\Lambda(m,:)\|_{0}^{2}\right|_{+1)=\mathrm{i}}\right. \\
& p(i)=\frac{\gamma_{m} \times \sigma^{2}}{\left|h_{i, m}\right|^{2}}
\end{aligned}
$$

end

$$
\begin{aligned}
& \text { for } n=1: N_{\max } \text { do } \\
& \mid \begin{array}{l}
\text { for } l=1:|\mathbb{C}| \text { do } \\
\left.\right|_{\text {end }} \mid \operatorname{lf}(\Lambda(n, l) !=0) \text { then } \\
\text { end }
\end{array}
\end{aligned}
$$

Proposition 2. The power allocation schema, resulting from Algorithm [1] is a Nash equilibrium for the proposed power control NOMA based game.

Proof. Assume that an MTD would like to change to a higher SNR. This could happens only if a new MTD j joins the same target SNR $\Gamma$ decreasing the transmission probability of MTD $i$. Assume MTD $i$ would prefer to target SNR $\Gamma^{\prime}$. Since MTDs are sorted according to their channels, if MTD $i$ can target $\Gamma^{\prime}$, $j$ can target it also and thus $j$ will not target $\Gamma$ since $\Gamma^{\prime}$ gives better utility, which leads to a contradiction. We can prove analogously that an MTD has no incentive also to decrease its target SNR. Hence, since no player can deviate from the power allocation schema, the proposed algorithm achieves the Nash equilibrium.

\section{B. Hedonic game coalition formation algorithm}

In this section, we focus on how MTDs arrange themselves into clusters using a hedonic game.

Definition 1. A hedonic game $\left\langle\mathcal{N}, \succeq_{i}\right\rangle$ is formulated by a finite set of devices $\mathcal{N}$, and a set $\left\{\succeq_{1}, \succeq_{2}, \cdots, \succeq_{N}\right\}$ of preference profile, which specifies a preference relation for each player $i \in \mathcal{N}$, a reflexive, complete and transitive binary relation on set $\mathcal{N}_{i}=\{\mathbb{S} \subseteq \mathcal{N}|\rangle \in \mathbb{S}\}$.

\footnotetext{
${ }^{2}$ is the $L_{0}$ norm, which is the number of non-zero elements.
} 
We say that a coalition $\mathbb{S}$ is preferred to MTD $i$ than coalition $\mathbb{S}^{\prime}$ if $U_{i}(\mathbb{S} \cup\{i\}) \geq U_{i}\left(\mathbb{S}^{\prime} \cup\{i\}\right)$. We denote by $\sim_{i}$ and $\succ_{i}$ the indifference relation and strict preference relation of an MTD $i$ respectively. Since an MTD evaluates his preference over his coalition and not on the whole structure, the considered coalition formation game is called hedonic game with a Non-Transferable Utility (NTU).

Definition 2. A partition denoted $\psi=\left\{\mathbb{C}_{1} \ldots \mathbb{C}_{k}\right\}$, is a set of a number of subsets of $M$ involving all players of $M$.

\section{1) Stability:}

In the hedonic games literature, we have identified the following stability concepts: Nash stability, individual stability, contractual individual stability and core stability. The core stability requires the immunity to coalition deviation, where in the other ones, a partition is stable when it is immune to individual deviation. Specifically, a partition is Nash stable if no MTD can improve his utility by moving unilaterally to another existing coalition. On the other side, a partition is individually stable if there is no MTD who can improve his utility by moving to another existing coalition $\mathbb{S}^{\prime}$ (by creating a new coalition eventually) without making the members of $\mathbb{S}^{\prime}$ worse off. Similarly, a partition is said to be contractually individually stable if there is no MTD who can improve his utility by moving from a coalition $\mathbb{S}$ to another existing coalition $\mathbb{S}^{\prime}$ (by creating a new coalition eventually) without making the members of $\mathbb{S}$ nor the members of $\mathbb{S}^{\prime}$ worse off. Finally, a coalition $\mathbb{S} \subseteq \mid \mathcal{N}$ is said to block a partition $\mathcal{M}$, if each MTD of coalition $\mathbb{S}$ strictly prefers $\mathbb{S}$ to his current coalition in $\mathcal{M}$. If there is no blocking coalition, the partition $\mathcal{M}$ is said to be core stable.

\section{2) Algorithm for generating Nash stable coalition struc-} tures:

Algorithm 2 illustrates the formal description of our algorithm. Firstly, an MTD joins the cluster corresponding to his best channel (lowest transmit power). Then, he determines his power allocation coefficient and transmission probability by executing the proposed Power Control Algorithm (Algorithm 11. Second, each device aims to maximize his utility, so that he invokes the Algorithm 11 at each iteration and anticipates his new utility if he deviates from his current coalition to another one. If devices are no longer interested to leave their groups and switch to other groups, the algorithm terminates. A summary of the proposed algorithm is given in Algorithm 2 Let us focus now on the stability of the proposed algorithm.

Theorem 2. The partition resulting from Algorithm 2 is Nash Stable.

Proof. Since the algorithm terminates when there is no incentive for any user to leave his current coalition, we deduce from the definition of the stability that the proposed partition is Nash Stable.
Algorithm 2 Nash stable coalition structures generation NSCSG

Input: the set of devices in the cell $\mathbb{M}$ and their channels $\mathbf{H}$, the number of RB $K$

Output: a partition $\Psi$

Initialization: $\Psi=0_{K \times|\mathbb{M}|}, U=0_{1 \times|\mathbb{M}|}$

every MTD sorts $(\mathbf{H}(i,:), i)=\left(h_{i, 1}, \ldots, h_{i, K}\right)$ using first coordinate in a decreasing order obtaining vectors $\left(h_{i, 1}^{\prime}, \ldots, h_{i, K}^{\prime}\right)$ and $\Pi_{i}$

for $i=1:|\mathbb{M}|$ do

find first $j$ such that $\boldsymbol{\Psi}\left(\Pi_{i}(1),:\right)=0$

$\boldsymbol{\Psi}\left(\Pi_{i}(1), j\right)=i$

end

for $k=1: K$ do

$$
\begin{aligned}
& {[\mathbf{p}, \mathbf{p t}]=P C(\mathbf{\Psi}(k,:), \mathbf{H}(k, \mathbf{\Psi}(k,:)))} \\
& \text { for } i=1 \in \mathbf{\Psi}(k,:) \mathbf{d o} \\
& \quad U(i)=p t(i) \frac{f\left(\gamma_{t h}\right)}{p(i)} \\
& \text { end }
\end{aligned}
$$

end

isFinal $=$ False

while !isFinal do

isFinal=True

for $i=1:|\mathbb{M}|$ do

for $k=1: K$ do

if $i \notin \mathbf{\Psi}(k,:)$ then

$\mathbf{h}_{t m p}=\mathbf{H}(k, \mathbf{\Psi}(k,:)) \cup h_{i, k}$

$[\mathbf{p}, \mathbf{p t}]=P C\left(\mathbf{\Psi}(k,:), \mathbf{h}_{t m p}\right)$

if $p t(i) \frac{f\left(\gamma_{t h}\right)}{p(i)}>U(i)$ then

$U(i)=p t(i) \frac{f\left(\gamma_{t h}\right)}{p(i)}$

$\mathbf{\Psi}=\mathbf{\Psi} \backslash\{i\}$

find first $j$ such that $\boldsymbol{\Psi}(k,:)=0$

$\boldsymbol{\Psi}(k, j)=i$

isFinal $=$ False

end

end

end

end

end

\section{Simulation Results}

In this section, we present a comprehensive Matlab-based simulation of the proposed bi-level game, described in the previous sections, for channel selection and power allocation in NOMA network. We consider the slotted Aloha based NOMA, introduced in [6], as a reference technique to compare with. We consider a scenario composed of $M=600$ MTDs independently deployed within a cell of radius $r=100 \mathrm{~m}$ according to homogeneous PPP process of density $\lambda_{M}=0.01$.

\section{A. Packet transmission rate with success}

In this section, we focus on the probability that a packet is transmitted successfully using the proposed bi-level game theoretical technique versus slotted Aloha based NOMA. As 


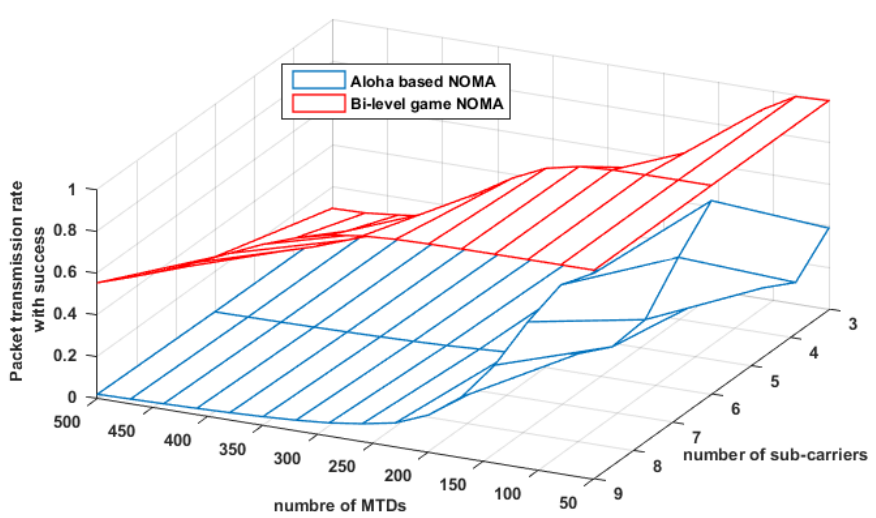

Fig. 2. The packet transmission rate with success.

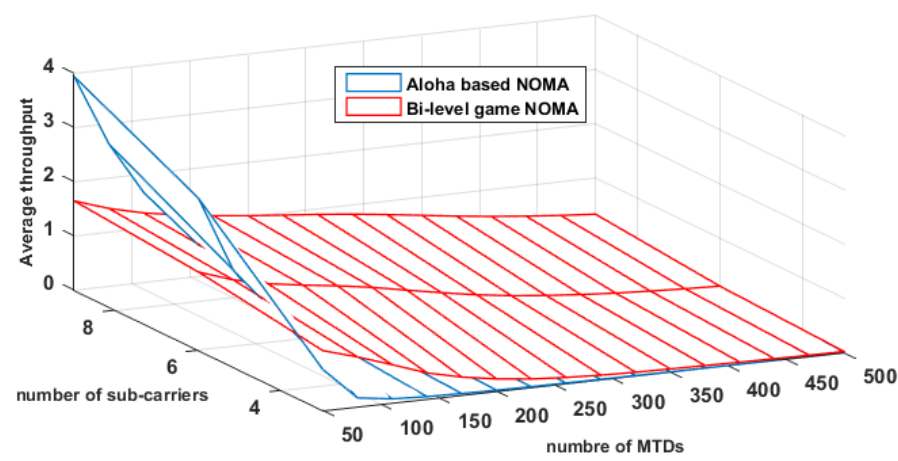

Fig. 3. The average throughput for the proposed model vs Aloha based NOMA.

we can observe in Figure 2, the packet transmission rate with success decreases drastically for slotted Aloha based NOMA, due to collisions, while the proposed technique achieves very good performances. For example, when we have $K=9$ sub-carriers and $M=290$ MTDs, the packet transmission probability with success is 0.0622 for slotted Aloha and 0.9634 for the proposed technique. Note that the collisions not only decrease the system throughput, but also increase the energy consumption of the WSN.

\section{B. Average throughput}

This section is devoted to performance comparison of the proposed joint channel selection and power control technique versus the slotted Aloha based NOMA. Figure 3 illustrates the average throughput for the proposed technique versus the baseline schema as a function of the number of sub-carriers and the number of MTDs in the WSN. As it was expected, the average throughput of the slotted Aloha is higher than the proposed technique when there is a low number of MTDs. In fact, Figure 4 illustrates that for $K=9$ sub-carriers, slotted Aloha achieves higher throughput for $M<140$ MTDs. This result is somehow intuitive, since the proposed technique allocates the minimal transmit power required to

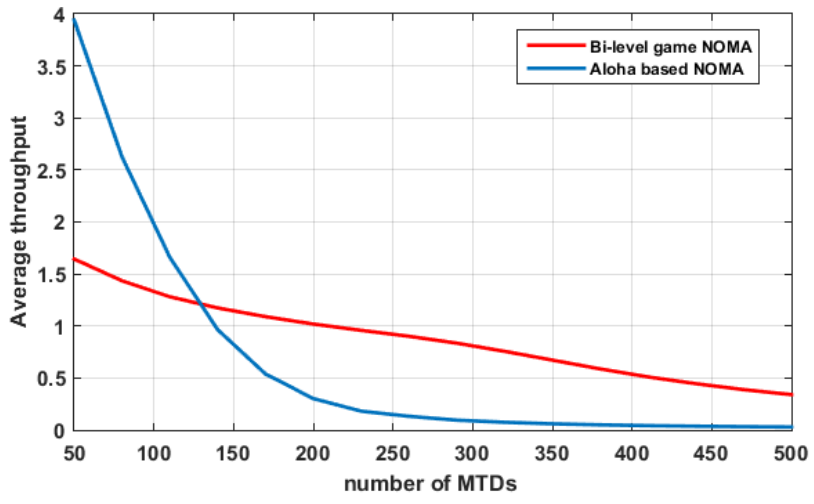

Fig. 4. The average throughput for $K=9$ sub-carriers.

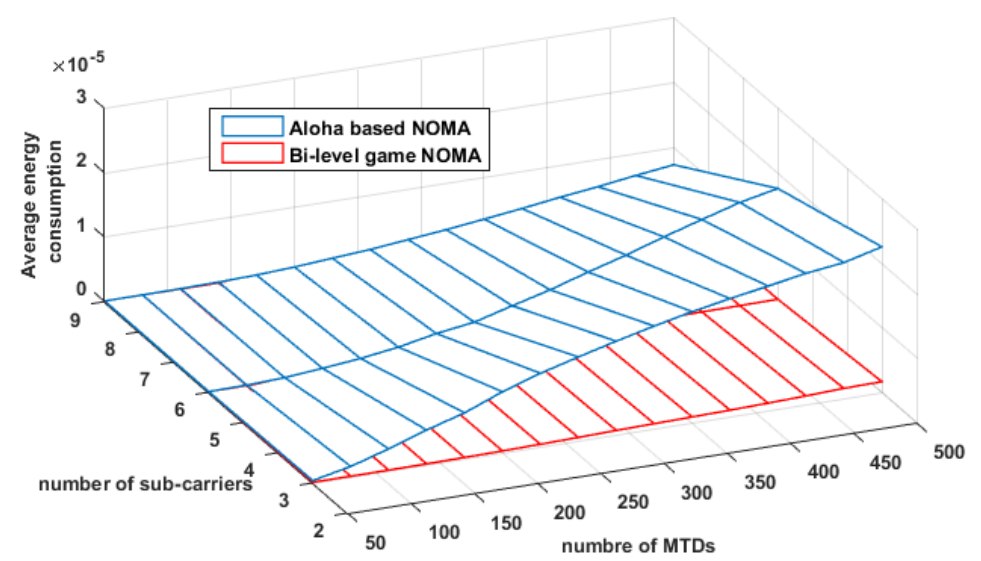

Fig. 5. The average energy consumption.

deliver the packet, while slotted Aloha may allocate to MTDs higher transmit power, and thus higher throughput. Since IoT devices require high reliability and low data-rate, the proposed technique is very well suited for the IoT scenario.

\section{Energy consumption}

Finally, We use a simplified model [15] for the energy consumption. Hence, the required energy for an MTD to send an L-bit packet is given by

$$
\begin{cases}L \times E_{\text {elec }}+L \times \epsilon_{f s} d^{2} & \text { if } d<d_{0} \\ L \times E_{\text {elec }}+L \times \epsilon_{\text {amp }} d^{4} & \text { if } d \geq d_{0}\end{cases}
$$

where $E_{\text {elec }}$ is the electronic energy. By comparing the distance $d$ between MTD and BS to the threshold distance $d_{0}$, either the free space $\epsilon_{f s}$ ( $d^{2}$ power loss) or multi path fading $\epsilon_{a m p}\left(d^{4}\right.$ power loss) channel model are used. For making this paper self-contained, we review here the above parameters from [15] in Table 1 .

As we can clearly see in Figure 5, the MTDs consume far less energy when using the proposed technique than the conventional slotted aloha technique. This energy efficiency 
makes the proposed technique as an interesting candidate to tackle the integration of IoT into beyond $5 \mathrm{G}$ systems.

TABLE I

ENERGY CONSUMPTION PARAMETERS

\begin{tabular}{|l|l|l|}
\hline Symbol & Description & Value \\
\hline$d_{0}$ & Threshold distance $(\mathrm{m})$ & 87 \\
\hline$E_{\text {elec }}$ & Electronic energy (nJ/bit) & 50 \\
\hline$\epsilon_{f s}$ & $\begin{array}{l}\text { Power amplification for the free space } \\
(\mathrm{pJ} / \mathrm{bit} / \mathrm{m} 2)\end{array}$ & 10 \\
\hline$\epsilon_{a m p}$ & $\begin{array}{l}\text { Power amplification for the multi-path } \\
\text { fading (pJ/bit/m2) }\end{array}$ & 0.0013 \\
\hline
\end{tabular}

\section{CONCLUSION}

In this paper, we have derived two-low complexity algorithms for MTDs in hybrid NOMA based on bi-level game theory. First, the MTDs self-arrange into coalitions using the hedonic game framework. Then, the members of each coalition invoke non-cooperative NOMA-based PC game to determine the transmit powers to use for sending their packets over one $\mathrm{RB}$ and in a non-orthogonal manner. The provided simulation results demonstrate that our proposed scheme enables the MTDs to meet the SINR requirement with less energy consumption compared to the conventional slotted aloha. In other words, our proposed bi-level game has efficiently solved the joint channel selection and power allocation while striking an attractive trade-off between system capacity and energy consumption. Therefore, simulation results have proved the efficiency of our proposed technique compared to the slotted Aloha NOMA schema.

\section{REFERENCES}

[1] C. Liu and D. Liang, "Heterogeneous networks with power-domain noma: Coverage, throughput, and power allocation analysis," IEEE Transactions on Wireless Communications, vol. 17, no. 5, pp. 35243539, May 2018.

[2] W. Han and X. Ma, "Power division multiplexing," in 2016 IEEE/CIC International Conference on Communications in China (ICCC), July 2016, pp. 1-6.

[3] B. Di, L. Song, Y. Li, and S. Zhang, "Trellis coded modulation for code-domain non-orthogonal multiple access networks," in 2018 IEEE International Conference on Communications (ICC), May 2018, pp. 16.

[4] M. Gan, J. Jiao, L. Li, S. Wu, and Q. Zhang, "Performance analysis of uplink uncoordinated code-domain noma for sins," in 2018 10th International Conference on Wireless Communications and Signal Processing (WCSP), Oct 2018, pp. 1-6.

[5] M. S. Ali, H. Tabassum, and E. Hossain, "Dynamic user clustering and power allocation for uplink and downlink non-orthogonal multiple access (noma) systems," IEEE Access, vol. 4, pp. 6325-6343, 2016.

[6] J. Choi, "Noma-based random access with multichannel aloha," IEEE Journal on Selected Areas in Communications, vol. 35, no. 12, pp. 2736 2743, 2017.

[7] N. Zhang, J. Wang, G. Kang, and Y. Liu, "Uplink nonorthogonal multiple access in 5g systems," IEEE Communications Letters, vol. 20, no. 3, pp. 458-461, March 2016.

[8] J. Choi, "Joint channel selection and power control for noma: A multiarmed bandit approach," , MOMENT Workshop, IEEE PIMRC, Apr 2019.

[9] H. Jiang, Q. Cui, Y. Gu, X. Qin, X. Zhang, and X. Tao, "Distributed layered grant-free non-orthogonal multiple access for massive mtc," in 2018 IEEE 29th Annual International Symposium on Personal, Indoor and Mobile Radio Communications (PIMRC), Sep. 2018, pp. 1-7.
[10] M. Haddad, P. Wicek, O. Habachi, and Y. Hayel, "On the two-user multi-carrier joint channel selection and power control game," IEEE Transactions on Communications, vol. 64, no. 9, pp. 3759-3770, Sep. 2016.

[11] Kaidi Wang, Z. Ding, and Wei Liang, "A game theory approach for user grouping in hybrid non-orthogonal multiple access systems," in 2016 International Symposium on Wireless Communication Systems (ISWCS), Sep. 2016, pp. 643-647.

[12] Z. Ding, P. Fan, and H. V. Poor, "Impact of user pairing on 5g nonorthogonal multiple-access downlink transmissions," IEEE Transactions on Vehicular Technology, vol. 65, no. 8, pp. 6010-6023, Aug 2016.

[13] A. Goldsmith, Wireless Communications. New York, NY, USA: Cambridge University Press, 2005.

[14] F. Meshkati, Mung Chiang, H. V. Poor, and S. C. Schwartz, "A gametheoretic approach to energy-efficient power control in multicarrier cdma systems," IEEE Journal on Selected Areas in Communications, vol. 24, no. 6, pp. 1115-1129, June 2006.

[15] Z. Li, Y. Liu, M. Ma, A. Liu, X. Zhang, and G. Luo, "Msdg: A novel green data gathering scheme for wireless sensor networks," Computer Networks, vol. 142, pp. 223-239, 2018. 